said that he had never thought of that. Dr. Marcy advised him, on his return, to have all these patients examined carefully and to report results. Over one hundred of these women were operated on for serious pelvic disease, and over 70 per cent. were permanently restored to their normal mental condition.

Dr. G. B. MAsser, Philadelphia, called attention to the cataphoric diffusion through the vagina of mercuric ions, in inflammation in the pelvis. It does not matter whether this inflammation is in the uterus, in the tubes, in the ovaries or in the parametrium, it is still inflammation. There is something between these two alternatives of pelvic disease and psychosis that must be considered at times, and that is abdominal neurosis, not psychosis. Eliminate hysteria, exclude psychosis, and though hysteria is a manifold complex disease, it can seldom be mistaken, but do not treat cases of abdominal neurosis as hysterics. Do not put them in bed; it will make them worse. Correct the abdominal condition. Decide in every case the output of solids in the urine. See that there is no kidney constipation, and many of these cases will recover.

DR. D. H. Craig, Boston, said that in examining the ovaries of young women more healthy looking ovaries are found than in women a little older who have had nearly as smooth a life as the young women. On investigating the subject, it occurred to him that the ovary of a woman comparatively recently entered on her menstrual life is relatively sound, whereas the ovary of a woman who has menstruated for some years has a scar for each time an ovum has ripened and the follicle ruptured. At the middle or end of menstrual life, therefore, the ovary is a much scarred, contracted organ. In the average normal ovary there are from ten to eighteen graafian follicles in process of maturation, and many follicles attain a diameter of $2 \mathrm{~cm}$. Dr. Craig has seen many a badly scarred ovary which gave no clinical symptoms but which was so apparently cystic that he put a cautery point or knife into the sacs of fluid and evacuated a great deal of material, dropped it back, and the woman went on comfortably. The question arises whether those scars were not the result of previously ruptured follicles and whether the "cysts" he evacuated were not maturing graafian follicles. After removing such ovaries, he has had them examined microscopically and in some instances his suspicions were confirmed. Many of the so-called cystic ovaries removed to-day; that have given rise to no clinical symptoms of ovarian disease, are not pathologic ovaries at all but are ovaries containing graafian follicles which are rather larger than common but are simply in process of maturation.

Dr. Cokenower does not believe that any one who has not been a general practitioner first should be a specialist in any line of work, especially gynecology, a fact that his consultation work with general practitioners and other gynecologists has verified. Physicians are too prone to limit their knowledge to their own subject, thus excluding much that is valuable in other subjects; hence, their observations should be more general and their work broader, thus producing better results.

\section{MANAGEMENT OF THE ACUTE STAGES OF ABDOMINAL INFI_AMMATION.*}

GEORGE ERETY SHOEMAKER, M.D.

Gynecologist to the Presbyterlan Hospital.

\section{PHILADELPHIA.}

Differential diagnosis is of the first importance, and will first engage the attention of the surgeon. This paper is concerned with the management of acute stages after the diagnosis is made, especially with the question of immediate operation, and only acute conditions will be considered. It may be necessary, clinically, to distinguish the following: Suppurative cholangitis;

* Read at the Fifty-fifth Annual Session of the American Medical Association, in the Section on Obstetries and Diseases of Women, and approved for publication by the Executive Committee : Drs. J. H. Carstens, A. Palmer Dudley and L. H. Dunning. suppurative cholecystitis associated or not with gallstones; acute suppurative pylephlebitis; various kidney inflammations; peritonitis following the perforation of viscera or of cystic tumors; acute hemorrhagic pancreatitis; ureteral calculus. Among intestinal conditions may be found strangulated hernia: obstruction due to bands; intussusception; volvulus of mesentery or of intestine; acute appendicitis; thrombosis or embolism of mesenteric vessels or those of the spleen. Salpingitis, ovaritis, metritis; postpuerperal, traumatic or due to such infecting agents as the tubercle bacillus or the gonococcus.

Of these conditions certain ones do not call for operation, and are, therefore, to be distinguished. Acute suppurative pylephlebitis, for example, the septic inflammation of the veins of the liver, is an invariably fatal disease. Fortunately, it is usually secondary to an infection along the portal tract, and is, therefore, preventable. A case reported by me was due to acute appendicitis, and another to postperitoneal infection caused by a common pin in the appendix. The primary condition might call for operation, but not the secondary, as the hopeless multitude of liver abscesses and the widespread engorgement of veins by virulent thrombi make radical treatment or even drainage impossible.

Extensive thrombosis of the mesenteric vessels and spontaneous gangrene of the intestine is beyond operative relief, but inasmuch as the area involved may be small enough to make resection feasible, exploratory operation would be indicated were the diagnosis made. It shares with acute pancreatitis certain crucial symptoms. Very sudden agonizing pain, usually above the umbilicus, vomiting, collapse and muscle rigidity. There may also be copious, loose, bloody or coffee-ground stools. The pulse is high and meteorism is very marked. Should the probable diagnosis of acute pancreatitis be made, there is some hope from early operation and thorough drainage. Osler mentions two cases which recovcred after partial or total sequestration of the pancreas within an abscess cavity. The mistaken diagnosis of acute intestinal obstruction is very apt to be made in these cases.

Should acute necrosis of the spleen occur from occlusion of its blood supply operative extirpation would be probably useless, but theoretically it would be indicated.

There is a simulation of abdominal inflammation which deserves to be carefully considered, namely, pain referred to the epigastrium or appendix region in the early stages of pneumonia. In most of these rather puzzling cases, however, the real origin of the disease is in the appendix, and the chest symptoms are due to a secondary diaphragmatic pleurisy or to a septic pneumonia. When the onset has been distinctly abdominal, the later development of lung symptoms must not be allowed to befog the diagnosis of appendicitis. We come, then, to a group of conditions in which the treatment, at least, of all the graver types is operative, but in which the indications are to avoid operation in the acute stage, if possible, relieving by simple drainage if compelled to intervene. Operate radically after the primary stages of infection are past. To this type belongs salpingitis in all its forms, gonorrheal, traumatic, tubercular, post-puerperal ; also cholecystitis, except the fulminating or gangrenous forms. The gall bladder is. however, like the appendix, much more liable to the treacherous, rapidly fatal conditions of profound infection 
and will not bear at all a management which follows set rules and does not allow of variations.

There remains, then, by far the largest group, namely, that in which operation is indicated at the earliest possible moment of an acute onset. Appendicitis is here incluled, with some modifications in cases first seen in later stages, also intestinal obstruction, perforation of all viscera, and strangulated hernia. After perforation of any of the viscera, immediate and radical operation is indicated as soon as the diagnosis can be made, or, in severe cases, with very suspicious symptoms, even before the diagnosis is certain. The repair is to be made with as little handling of bowel as possible, the peritoneal cavity being carefully cleaned by sponging if the affected area is small, or by copious irrigation if there are no limiting adhesions and contamination is extensive. Drainage by tube and gauze follows, of the region affected and of dependent points.

Strangulated hernia, or inflamed irreducible hernia, both call for immediate operation, day or night. $\mathrm{Pa}$ tients with such disorders do not bear transportation well, especially if advanced in years. It is often wiser to operate in the patient's home than to invite disaster by transporting him to a hospital. Space forbids here any consideration of operative technic. The truest conservatism almost always calls for the total removal of the appendix as contrasted with a drainage operation in acute attacks. Gonorrheal tubes, if operated on at all, should both be removed into the cornu of the uterus.

\section{MEDICINAI 'TREATMENT.}

In cases where it has been decided not to operate, vomiting is best controlled by withholding all food, and, if necessary, by lavage of the stomach. Feeding by the rectum sustains the strength and rests the disordered digestive tract. Tympany may be relieved by hypodermic injection of salicylate of eserin, 1/100 gr., among other measures. Normal salt solution by the rectum or under the skin flushes the kidneys, relieves thirst, sustains the pulse and assists in the elimination of toxins.

The ice bag is probably the most useful local agent we have. Ichthyol may be applied to the skin under it. Flannel should always intervene to take away the sense of chill. My custom is not to use ice continuously, but two hours out of every three. Local sloughs have been reported as caused by its continuous use.

Opium in acute conditions is dangerous, because it masks the symptoms and has thus caused many deaths.

Acute cholecystitis, whether due to gallstone disease or to infection by specific organisms, such as the typhoid bacillus, may be cautiously watched through acute stages with everything ready for immediate interference if called for. Gangrene and perforation are not common, but they call for immediate operation at any time when they may be discovered.

\section{APPENDICITIS.}

Next to inflammatory disease of the pelvic organs in women, quite the most frequent cause of acute abdominal inflammation is appendicitis. As is well known, the pain and tenderness may be referred to the epigastrium or liver region, to the pelvis, or even to the left side of the lower abdomen. Should a patient have sudden severe pain in these regions, with chilliness, vomiting, a little fever and an anxious expression of the face, with muscular rigidity over the right abdomen in the lower quadrant, appendicitis is probably present. No matter where the pain may be referred, the muscular rigidity is always over the diseased organ.
When the attack of acute appendicitis is serious and decided, there is general agreement that the appendix should be at once removed. No time should be lost in obtaining a blood count, which can only corroborate what the clinical picture teaches. If taken, however, it will probably show a leucocytosis above 15,000 , with a tendency to increase; but a high white cell count alone is not a good guide for or against operation, though it furnishes valuable corroborative evidence. Its absence must not be too highly regarded if other symptoms of gravity are present, especially an unsatisfactory pulse and a facies which shows anxiety, associated with sallowness or slight jaundice and duskiness. In profound toxemia the facial expression may be dull and listless.

Micro-organisms.-The organisms most frequently met in abdominal inflammations are the gonococcus, the colon bacillus, the streptococcus, the tubercle bacillus and staphylococci. The typhoid bacillus is occasionally found in pure culture in the gall bladder, as in one of my cases.

However difficult it may be to demonstrate it in the laboratory, there are undoubtedly stages of growth in which organisms vary greatly in their power to produce toxemias. Widespread as the colon bacillus is, it is only in some stages that it forms a highly dangerous infective agent. For example, in gunshot wounds of the healthy intestine, these bacilli escape into the peritoneal cavity without doing harm, if reasonably prompt suture of the openings and the toilet of the peritoneum is carried out. A similar amount of implantation of the same bacillus as it appears when migrating through the walls of a gangrenous volvulus gives rise to a rapid form of infection after an equivalent number of hours' residence in the peritoneum, which no amount of irrigation or cleansing is able to offset.

The comparison intended is the same as that which may be made between the organism of diphtheria demonstrable in the discharges of ozena, as compared with the same organism appearing in the infectious stages of true-diphtheria. The same difference holds good in regard to stages of virulence in streptococci and the pusforming organisms.

The deduction is this, that under the conditions which obtain when the bowel wall is acutely inflamed, especially where the blood supply is suddenly cut off, virulent states of previously benign organisms rapidly develop.

Most important in the case of appendicitis is the concealment under which this process at times proceeds. A history such as this is well known in the experience of every operating surgeon. A patient has appeared per fectly well till within thirty-six or forty-eight hours of the time he is brought for operation. For the first twenty-four hours of that time symptoms have been so mild as not to confine him to bed, and to his friends, or even to his physician, there has seemed little cause for anxiety. The temperature was not above 99 , and there was little pain, though for a few hours in the beginning it had been severe. Only the pulse was suspiciously high. Twelve hours later the patient's condition had obviously changed for the worse; the pulse was 120 to 130 and feeble, the tongue dry, the face anxious and slightly jaundiced, or with a sickly pallor. In such a case the temperature may be from 99 to 101 . Tenderness of the abdomen may be slight or severe, according to whether the entire area involved is gangrenous, or whether surrounding peritoneal areas are only inflamed. The skin becomes leaky, the pulse thready, rapid and running, the voice weak. The subcutaneous capillaries 
are irregularly distended; there are mottled bluish patches about the abdomen and thighs; the mind, at tirst clear, becomes dull. The patient, who seemed so well after the first twenty-four hours, is at the end of forty-eight hours on the verge of the grave.

What has happened here is that profound toxemia has developed quietly, while the absence of marked pain or extreme degrees of temperature, vomiting or tympany has given the attendants a false sense of seeurity. There may or may not have been a rupture of the appendix. There has been here the development of a highly virulent condition of the ordinary bacilli of the intestine. 'The products which accompany their growth are intensely poisonous. Absorption has taken place through portal vein radicles and has reached the liver, or postperitoneal lymphatic channels have poured it into the great lymph streams. Whatever the route, the toxic dose received by the patient was sudden and overwhelming. In spite of operative removal of the appendix and drainage at this time, early in days but late in infection, there is little hope for the patient. It is the treacherous character of infection of the appendix, its power of remaining latent or concealed, and bursting forth into an overwhelming toxemia, that furnishes the keynote for the management of the disease.

If seen early, operate early and radically, in all except the simplest cases, without waiting for dangerous symptoms, when it may be too late. The management of late stages, seen only when late, presents special problems.

INFLAMMATORY CONDITIONS IN THE PELVIS.

The second most formidable infecting organism met with in acute conditions is the streptococcus. Especially as found in post-puerperal inflammations, it gives rise to many fatalities. The cardinal principles of management of such infections may be briefly summarized as follows: Avoid, if possible, opening the general peritoneal cavity. Thoroughly cleanse the vaginal and uterine field by gentle mechanical removal of débris without abrading fresh surfaces. Use tincture of iodin and loosely pack uterus and vagina with iodoform gauze.

If the conditions be not promptly arrested, open the vaginal cul-de-sac and isolate the uterine field by iodoform gauze, as advocated by Pryor. Should pus form in the pelvis, every effort should be made to drain it from the vaginal side to tile over the acute stages. Operate radically weeks or months later, if necessary, when much of the danger of peritoneal operative infection will have passed away. Acute symptoms, due to tuberculosis of the tubes and ovaries or of the urinary tract, are, if severe, apt to be due to mixed infection. It is wiser not to operate radically in the height of an inflammatory attack, but to tide over the acute period by ice externally and general measures, including good care and nursing. Pus is to be immediately drained away, for example, from the kidney, reserving nephrectomy for a later period.

Gonorrhea is the most common, and to life least dangerous, of the causes of acute abdominal inflammation, especially in women. Its results in producing permanent ill health are, however, appalling, and many deaths follow more or less indirectly. Immediately after a menstrual period, or immediately after childbirth, a fresh infection appears more liable than at other times to spread to the peritoneum and to give rise to serious lesions.

In the acute stage prompt attention will greatly modify the destructive process. Rest in bed, liquid diet, saline laxatives, external cleanliness, gentle irrigation with lysol or potass. permanganate, 5 per cent. protargol solution to the urethra, will promptly ameliorate the external symptoms, and while these measures do not cure the tubal conditions, they lessen the tendency to reinfection and extension. Prompt amelioration of pain, temperature and tenderness should follow. According to Pryor, opening the vaginal cul-de-sac and packing the pelvic cavity with properly prepared iodoform gauze will prevent serious lesions of the tubes in nearly all cases if done promptly. Radical extirpating operations should never be done in the acute stages, especially of first attacks. Too long delay, however, may be worse, and if pus forms in quantity it must be evacuated by the nearest route; otherwise it may burrow or perforate into bowel or bladder. Gonorrheal cases are not favorable for partial operations, which aim to preserve organs. No greater contrast in the management of acute conditions exists than between that of acute appendicitis and acute gonorrheal salpingitis, or salpingitis due to cold or dampness while menstruating. In appendicitis, owing to the treacherous nature of the disease, operation is usually safer than waiting, and, except in the simpler cases, it should be done as early and as completely as possible, being followed by free drainage. The appendix can be removed in all but the most exceptional cases, and it should be done.

In salpingitis all palliative measures should be employed during the acute stage, and operation never undertaken then except for good reason. If necessary to drain a large pus collection, do it preferably by the vagina. In gonorrhea a few cures will be accomplisherl in this way, though the mortality of the subsequent major operations will be greatly lessened. It is possible thus to handle a series of pus cases with a mortality of 4 or 5 per cent. As bad pus cases formerly constituted the chief element in mortality in any series of unselecter cases, a great gain has been made by the adoption of this plan of management, so that it is now possible to keep a general operating mortality well under 6 per cent. and to have a long series of cases without a death. The mortality in the simpler chronic gonorrheal salpingitio cases is almost nothing.

\section{REMARKS.}

No greater mistake can be made, however, than to make these rules apply rigidly to all cases. The surgeon must ever be on the alert for signs which call for immediate operative interference.

Of danger signals, the most important is the pulse. A rate high in proportion to the severity of other symptoms is very suspicious and should lead to the utmost vigilance. An ascending rate with a diminishing quality means danger, and this no amelioration of other symptoms should outweigh. The temperature is an unsafe guide in abdominal inflammation, as the gravest lesions may be accompanied by little rise and a fall may be deceptive. A simultaneous and continued rise of both pulse and temperature is always unfavorable. Leucocytosis above 15,000 with a tendency to rise usually points to operation. A decided fall in a patient evidently very ill is not a good sign. Other unfavorable signs are persistent vomiting, increasing tympany, sweating, thoracic respiration, increasing muscular rigidity, failure of peristalsis, local edema. Pain generally improves with the patient's condition. A sudden cessation in an otherwise ill patient may point to gangrene.

1831 Chestnut Street. 


\section{DISCUSSION.}

Dr. JohN H. Fisher, Philadelphia, indorsed all that Dr. Shoemaker said on the management of acute inflammatory conditions in the abdominal and pelvic cavities, but emphasized that the tolerance of the peritoneum differs in various portions of the abdomen. It is more tolerant in the pelvis than in the upper portion. In acute inflammations in the pelvis acute symptoms should subside before operation. No risk should be run in appendicitis. The patient's life is not jeopardized by an early operation. In a gonorrheal case Dr. Fisher recently removed a large pus tube from one side. He thought of removing the other tube, but a physician present said that it was healthy and suggested that it be left. Dr. Fisher left it and the patient has since returned to have it removed. Gonorrhea in a female, although it may appear to involve only the appendage of one side at first, always becomes bilateral, and during an operation, when one side appears to be healthy, it is best to remove the tube, leaving, if possible, an uninvolved ovary.

Dr. F. B. Dorsey, Keokuk, Iowa, said that for a number of years it was his practice in gonorrheal patients to remove the tubes and to consider the patients cured, but on investigation he found that they have constant leucorrhea. For the last four or five years, whether the trouble is unilateral or bilateral, he removes the uterus also, and the results have been very satisfactory. He can not recall a single death from a gonorrheal inflammation during the acute stage, except those who were operated on by other men during the acute stage. The acute symptoms should subside before operation. If there is a mixed infection, drain through the vagina until the primary attack has subsided, and then do a radical operation. In mixed infection there is trouble outside of the tubes, involving, perhaps, the broad ligament or some of the other tis. sues. We are not yet able to differentiate between a return to the normal and gangrene; nor can we diagnose, in some cases, the presence of pus in or about the appendix. Within the last three months in four patients with normal temperature he found pus.

Dr. Charles L. Bonifield, Cincinnati, Ohio, declared it bad practice to remove both tubes and the uterus in every case because one tube is infected with the gonococcus. In the later cases he believes that one tube should be removed and the uterus curetted; part of the other tube and ovary may be removed and the woman will still be able to give birth to healthy children. In some cases, one appendage is removed and the woman is apparently cured of the gonorrheal discharge. Afterward she has another gonorrheal discharge and infection of the other tube. There is no proof that this came from the original infection, because one of two things is taking placeeither the woman is a prostitute and has been exposed to gonorrhea since the operation, or she is married and her husband has gonorrhea. Dr. Bonifield has seen a number of such cases where a woman was infected several weeks after coming from the hospital. Many of these instances of persistent discharge from the uterus are due to the fact that the removal of the tubes was imperfect. In the early days the tube was tied off anywhere near the uterus. Now, the careful surgeon will not only cut off the tubes close to the uterus, but he will dissect out the horns and thus prevent secondary infection. The inflammation inside the uterus can be cured by repeated curettage and prolonged local application. Gynecologists make a mistake when they curette and promise the woman she will be well. She should be instructed to come back to the office for inspection a few weeks after the curettage, and often it is necessary to continue the local applications.

Dr. Shofmaker said that in his service in the Presbyterian Hospital, Philadelphia, he had 83 consecutive operations with only one death. These included several cases of bad pelvic abscess, two from gonorrhea with gangrene of the intestine, just on the point of perforation; but both cases got well, although they would have died if they had not been operated on. Sometimes he removes the uterus and sometimes he does not, but the cornua should always be removed. Leaving one tube in the presence of the gonococcus is bad policy, unless there is a strong reason for making the effort, and we explain to the patient that she may require a secondary operation. He has tried the conservative method a number of times on gon. orrheal cases and has been disappointed. Almost invariably he had to do a secondary operation later. An uninvolved ovary will not very often become infected.

\section{ENURESIS IN CHILDHOOD.*}

From the Department of Pediatrics. University of Pennsylvania; Dr. J. P. Crozer Griffith, Physician in Chief.

\section{MAURICE OSTHEIMER, M.D.,}

Instructor in Pediatrics, Unjversity of Pennsylvania; Assistant Pediatric Physician and Physician to the children's Dispensary, University Hospital.

AND

I. VALENTINE LEVI, M.D.,

Assistant Pediatric Physician and Assistant Physician to the Children's Dispensary, University Hospital.

\section{PHILADELPHIA.}

Experience has taught that incontinence of urine, while not uncommon in adults, in women especially, is constantly noted in institutions for the treatment of children. During the past five years at the children's dispensary, University Hospital, we have found 85 cases of enuresis among 1,657 new patients treated, one out of almost every twenty children manifesting this condition, about 5 per cent. These figures tend to corroborate the statement made by Townsend ${ }^{1}$ that, when the families of children applying for treatment at the Children's Hospital, Boston, were questioned, 21.5 per cent. of all children were found to have or to have had enuresis. Out of 1,500 children, however, he found but 25 with incontinence of urine, about 1.6 per cent, while Adams $^{2}$ reported 55 cases out of 19,261 children, about .25 per cent. It is probable that more of the children whom we saw suffered from enuresis, although the limited history taken failed to elicit this fact. Adding to these five cases recently observed in private practice, we have the honor to present 90 cases of enuresis in children, detailing our plan of treatment.

The incontinence occurred at night only in 53 cases; was both diurnal and nocturnal in 35 cases; and was diurnal only in two cases. Forty-seven patients were boys and 43 were girls. In 59 children the enuresis persisted from infancy, called by Townsend "primary" cases; in 11 cases it was first noted at 3 years; in four at 4,5 and 6 years; in three at 9 years; in two at 7 years, and at 8 and 12 years, respectively, in one child, making 30 "secondary" cases. In one case the time of onset is unknown.

Twelve of the 90 children treated were of Jewish ancestry, six boys and six girls. All these boys were circumcised in infancy. Four other boys had also been circumcised, in only one of whom did the operation result in permanent recovery. Five children, four boys and one girl, made up an entire German family, two of the boys being twins. One boy was a Syrian, one girl Italian, and two boys were French; the others were all American born. The mother and six sisters of one boy were epileptics, and he also had epilepsy. It is interesting to note here that Trousseau ${ }^{3}$ believed enu-

* Read at the Fifty-fifth Annual Session of the American Medical Association, in the Section on Diseases of Children, and approved for publication by the Executive Committee: Drs. $\mathbf{S}$. W. Kelley, H. M. McClanahan and John C. Cook.

1. Archives of Pediatrics, 1887, vol. iv, p. 744

2. The Jotrnal A. M. A., 1885, vol. iv, p. 6.

3. Journal de Médecine et de Chlrurgie Pratiques, 1860, 2 s., จol. xxxl, p. 106 . 